\title{
Avant-propos de la première édition, 1986
}

Dans Vertigineuses symétries, je souhaite discuter les motivations esthétiques qui sous-tendent la physique du $X X^{\mathrm{e}}$ siècle. Je suis plus intéressé à transmettre au lecteur le sens du cadre intellectuel dans lequel opère la physique fondamentale que par l'explication du contenu factuel de la physique moderne.

Albert Einstein affirma un jour : " je veux savoir comment Dieu a créé ce monde. Je ne suis pas intéressé par tel ou tel phénomène, par le spectre de tel ou tel élément. Je veux connaître Sa pensée, le reste n'est que détail. »

Comme physicien, je suis tout à fait séduit par ce sentiment exprimé par Einstein. Alors que la majorité des physiciens se préoccupe d'expliquer des phénomènes spécifiques, ce qui est parfaitement légitime, un petit groupe de physiciens, les héritiers intellectuels d'Einstein, sont devenus plus ambitieux. Ils ont pénétré la forêt nocturne à la recherche du dessein ultime de la Nature et, dans leur hubris sans limite, ils affirment l'avoir entrevu.

Deux grands principes guident leur recherche : symétrie et renormalisabilité. La renormalisabilité décrit la façon dont sont reliés entre eux deux processus physiques dont les longueurs caractéristiques sont différentes. Tandis que je vais simplement évoquer la renormalisabilité, je me focaliserai sur la symétrie en tant que point de vue esthétique à travers lequel les scientifiques de la physique fondamentale voient la Nature.

La physique fondamentale a bénéficié d'un intérêt croissant au cours de ces dernières années. On ne compte plus les exposés sur la «nouvelle physique ». Aujourd'hui, la plupart d'entre nous ont appris qu'il existe des milliards et des milliards de galaxies, chacune contenant des milliards et des milliards d'étoiles. On nous a dit que le monde peut être compris en termes de particules subnucléaires, dont la plupart ne vivent que des milliardièmes de milliardième de seconde. Le lecteur informé a été étonné et ébloui. Oui, sans aucun doute, le monde de la physique moderne est merveilleusement bizarre. Des particules 
affublées de noms grecs gigotent au son de la musique quantique en mettant au défi le déterminisme classique. Mais, au bout du compte, le lecteur peut avoir l'impression qu'on lui a simplement fait ingurgiter des faits qui, aussi surprenants soient-ils, finissent par le faire bâiller d'ennui.

Ce livre s'adresse au lecteur intellectuellement curieux, qui veut aller au-delà des faits. J'ai une image mentale de ce lecteur : quelqu'un que j'ai connu dans ma jeunesse, quelqu'un qui est peut être devenu depuis architecte, artiste, danseur, courtier, biologiste ou avocat, quelqu'un d'intéressé par le cadre esthétique et intellectuel où se développe la physique fondamentale.

Cela ne veut pas dire que les découvertes étonnantes de la physique moderne ne seront pas expliquées dans ce livre. Elles devront nécessairement être expliquées avant que je puisse discuter utilement le cadre intellectuel de la physique moderne. J'espère néanmoins que le lecteur pourra bénéficier non seulement d'un clin d'œil complice à certains faits étonnants, mais avoir aussi l'intuition du cadre sans lequel ils resteraient simplement des faits.

Je n'ai pas essayé d'écrire une histoire détaillée et équilibrée des symétries en physique. Tout exposé dans lequel les développements majeurs peuvent être attribués à une poignée d'individus ne peut pas prétendre faire œuvre historique, et toute affirmation contraire doit être catégoriquement rejetée. En évoquant certains développements de la physique moderne des particules, l'éminent physicien Shelley Glashow a un jour remarqué : « les tapisseries sont le résultat d'un travail collectif de plusieurs artisans. Les contributions des différents artisans ne peuvent pas être discernées dans l'œuvre finale, les fils défaits ou incorrects ont été recouverts. Il en est ainsi de notre image de la physique des particules... [Le modèle standard] n'est pas apparu d'un seul coup dans le cerveau d'un seul physicien, ni même de trois. Ce modèle, également, est le résultat de l'effort collectif d'un grand nombre de scientifiques, à la fois expérimentateurs et théoriciens. » Et cependant, dans un exposé de vulgarisation comme celui-ci, je suis inévitablement obligé de simplifier l'histoire. Je suis confiant que le lecteur le comprendra.

Santa Barbara, avril 1986 